\title{
Plant stanol ester spreads as components of a balanced diet for pregnant and breast-feeding women: evaluation of clinical safety
}

\author{
K. Laitinen ${ }^{1,2} *$, E. Isolauri ${ }^{3,4}$, L. Kaipiainen ${ }^{5}$, H. Gylling ${ }^{6,7}$ and T. A. Miettinen ${ }^{5}$ \\ ${ }^{1}$ Department of Biochemistry and Food Chemistry, University of Turku, 20014 Turku, Finland \\ ${ }^{2}$ Functional Foods Forum, University of Turku, 20014 Turku, Finland \\ ${ }^{3}$ Department of Paediatrics, Turku University Central Hospital, Turku, Finland \\ ${ }^{4}$ Department of Paediatrics, University of Turku, 20014 Turku, Finland \\ ${ }^{5}$ Division of Internal Medicine, Department of Medicine, University of Helsinki, 00014 Helsinki, Finland \\ ${ }^{6}$ Kuopio University Hospital, 70211 Kuopio, Finland \\ ${ }^{7}$ School of Public Health and Clinical Nutrition, University of Kuopio, PO Box 1627, 70211 Kuopio, Finland \\ (Received 19 June 2008 - Revised 2 October 2008 - Accepted 15 October 2008 - First published online 19 November 2008)
}

Clinical safety of consuming plant stanol ester spreads during pregnancy and lactation, the impact on maternal and infant serum and breast-milk cholesterol and the ratios $(\mu \mathrm{mol} / \mathrm{mmol}$ of cholesterol) of synthesis and absorption markers were evaluated. Pregnant women $(n 21)$ were randomised to control and dietary intervention groups, the intervention including advice to follow a balanced diet and to consume spreads enriched with plant stanol esters. Participants were followed during and after pregnancy and their infants up to 1 year of age. A mean 1.1 (SD 0.4) g consumption of plant stanols during pregnancy and 1.4 (SD 0.9) g 1 month post-partum increased sitostanol and the markers for cholesterol synthesis, lathosterol, lathosterol/campesterol and lathosterol/sitosterol, and reduced a marker for cholesterol absorption, campesterol, in maternal serum. In breast milk, desmosterol was lower in the intervention group, while no differences were detected between the groups in infants' serum. Plant stanol ester spread consumption had no impact on the length of gestation, infants' growth or serum $\beta$-carotene concentration at 1 and 6 months of age, but the cholesterol-adjusted serum $\beta$-carotene concentration was lowered at 1 month in the intervention group. Plant stanol ester spread consumption appeared safe in the clinical setting, except for potential lowering of infants' serum $\beta$-carotene concentration, and was reflected in the markers of cholesterol synthesis and absorption in mothers' serum, encouraging further studies in larger settings.

Plant stanol esters: Pregnancy: Breast milk

Prevention is better than cure, particularly in CVD, which represents globally a leading public-health problem. These diseases are growing in magnitude and manifesting themselves at an ever earlier age. A central underlying dietary determinant is the intake of cholesterol and SFA ${ }^{(1,2)}$. The association between hypercholesterolaemia in women during pregnancy and the early onset of atherosclerotic lesions in their children ${ }^{(3)}$ underscores the need for early preventive measures. Furthermore, hypercholesterolaemia has been associated with pregnancyinduced hypertension ${ }^{(4)}$, thus compromising maternal health.

Total serum cholesterol rises up to $60 \%$ and the TAG concentration up to $150 \%$ with progressing pregnancy ${ }^{(5-7)}$. This is explained by hormonal changes as the concentrations of oestrogen and insulin increase ${ }^{(8)}$ and the enzymal activity of lipid-regulating lipoprotein lipase decreases ${ }^{(9)}$, securing a constant intake of energy for the fetus ${ }^{(10)}$. However, the rise in cholesterol concentration is not determined solely by hormonal changes but may also be regulated by diet ${ }^{(11)}$. In view of the potentially unfavourable health effects on both mothers and infants, an excessive rise in blood cholesterol may not be considered desirable. At particular risk are the mothers with elevated blood cholesterol, including those with familial hypercholesterolaemia, the condition of which is untreated during pregnancy.

Food products enriched with plant sterol and stanol esters limit the absorption of both dietary and endogenous cholesterol and have been shown to lower serum total and LDLcholesterol concentrations on an average by $10 \%$, with no effect on the HDL- cholesterol concentration ${ }^{(12)}$. Consumption of plant stanols as components of a balanced diet is considered safe in non-pregnant adults, since together with their natural daily dietary intake of approximately $15 \mathrm{mg} / 8400 \mathrm{~kJ} / \mathrm{d}$ in omnivores $^{(13)}$, their absorption from the gastrointestinal tract is minuscule ${ }^{(12)}$ and any possible concern over the absorption of fat-soluble vitamins may be overcome by sufficient dietary intake $^{(14)}$. However, based on the lack of research in the populations concerned, the consumption of plant stanols during pregnancy or before 5 years of age is considered improper.

The aim of the present pilot study is to evaluate the clinical safety of plant stanol ester spread consumption during pregnancy and breast-feeding, and the impact of intervention on serum and breast-milk lipid and sterol concentrations. 


\section{Methods}

\section{Subjects and study design}

Women were recruited to a maternal and infant nutrition study during the first trimester of pregnancy, initiated in 2002. Women in maternal welfare clinics in the city of Turku and neighbouring areas in South-West Finland were informed about the study by leaflets. Interested recipients contacted the research nurse, who gave further information on the study and scheduled their first visit to the study clinic in Turku University Central Hospital. Women were eligible for participation if they were at less than 17 weeks' gestation and had no metabolic or chronic diseases such as diabetes. At the first study visit, the baseline, they were randomised into control and dietary intervention groups. The intervention comprised counselling to follow a balanced diet recommended for pregnant women and provision of food products to support the achievement of the recommendations, whereas the women in the control group received no counselling or food products. In a sample of women $(n 21)$ and their infants, the present study evaluated the clinical safety of dietary intervention including consumption of plant stanol ester spreads and its impact on serum and breast-milk lipid and sterol concentrations.

The women from the intervention group ( $n$ 11) were included in the order of enrolment on the basis of the use of plant stanol ester spreads reported in their food diaries collected after initiation of the intervention. For each participant in the intervention group, a consecutive participant in the order of enrolment from the control group ( $n$ 10) was included in the analysis. The women were followed during each trimester of pregnancy and with the baby at 1, 6 and 12 months after delivery. The follow-up visits included measurements of heights and weights, while pre-pregnancy weight was self-reported. The women in both the intervention and control groups were of the same age (30.3 (SD 4.3) and 31.2 (SD 5.7) years in the intervention and control groups, respectively).

The weights and heights of the infants were measured, standardised for age and sex and thus expressed as height standard deviation (SD) scores and weight proportional for height (weight for height, \%) ${ }^{(15)}$, and their cognitive development was evaluated at 12 months of age using the Griffiths developmental scale ${ }^{(16)}$. Hospital records were examined for birth data and the duration of breast-feeding was recorded.

Fasting blood samples from women in the first $(13.5$ (SD 1.8) and 13.9 (SD 2.0) weeks of gestation in the intervention and control groups, respectively, $P=0.618$ ) and third (33.8 (SD 1.7) and 34.3 (SD 1.3) weeks of gestation in the intervention and control groups, respectively, $P=0.418)$ trimesters of pregnancy and 1 month post-partum and non-fasting blood samples from their infants at 1 and 6 months of age were drawn from the antecubital vein by a laboratory technician. Breast-milk collection was standardised for all mothers. At the beginning of the day, the infants were allowed to suckle for a few minutes and the mothers then obtained a milk sample by manual expression, after which feeding continued. All samples were frozen and stored at $-70^{\circ} \mathrm{C}$ until analysis.

Written informed consent was obtained from the participants and the study was approved by the Ethical Committee of the Hospital District of southwest Finland.

\section{Dietary intervention and assessment}

Dietary counselling for the women in the intervention group aimed to modify the dietary intake to comply with the currently recommended one ${ }^{(17,18)}$. Practical dietary advice was given, adjusted to the womens' current dietary habits and food diary analysis. The subjects were encouraged to increase their consumption of vegetables, fruits, wholegrain breads and cereals, to consume leaner meat products, low-fat cheese and milk products, and to use vegetable oil or soft margarine as spread and in food preparation. The advised diet would result in $55-60 \%$ of carbohydrates, $10-15 \%$ of protein and $30 \%$ of total fat of energy intake, MUFA contributing $10-15 \%$, PUFA $5-10 \%$ and SFA $10 \%$ or less of energy intake. Achievement of the recommended diet was supported by providing the participants in the dietary intervention group with food products available in the market with a favourable fat composition (e.g. plant stanol ester spreads, low-erucic acid rapeseed oil-based spreads and salad dressing) to be consumed at home. The products were provided until mothers discontinued exclusive breast-feeding. The women were given a recommendation on the amounts of the products to be used daily, providing $1.8 \mathrm{~g}$ per day of plant stanols from plant stanol ester spreads (Benecol ${ }^{\circledR}$ ). The consumption of plant stanol ester spreads $\left(\right.$ Benecol $^{\circledR}$ margarine and soft cheese) was recorded in 3-d food diaries including one weekend day, using household measures from which the intake of plant stanols (g) was calculated. The intake of stanols was 1.1 (SD 0.4) $\mathrm{g}$ in the third trimester of pregnancy and 1.4 (SD 0.9) g 1 month post-partum in women in the intervention group. None of the women consumed plant stanol ester products at the baseline, the first trimester of pregnancy. According to the calculations of daily intakes of energy and nutrients using the Micro-Nutrica ${ }^{\circledR}$ computerised program (version 2.5, Research Centre of the Social Insurance Institution, Turku, Finland), the dietary counselling resulted in changes in dietary intake attributable to a higher intake of unsaturated and a lower intake of SFA in the dietary counselling group, reported in detail elsewhere ${ }^{(19)}$. In the twenty-one women of the present study, no differences in the intakes of energy, energy-yielding nutrients, cholesterol, dietary fibre and fat-soluble vitamins between the intervention and control groups were detected (data not shown), except that the intake of vitamin $\mathrm{E}$ was $2.0(95 \%$ CI $0 \cdot 1,3 \cdot 8) \mathrm{mg}$ higher in the intervention group compared with the control group during the follow-up.

\section{Serum and breast-milk analysis}

Serum and breast-milk sterols and squalene were analysed by $\mathrm{GLC}^{(20)}$. Total cholesterol, squalene and non-cholesterol sterols were measured from the non-saponifiable material of serum by GLC using a $50 \mathrm{~m}$ long capillary column (Ultra-2, Agilent Technologies, Wilmington, DE, USA). The procedure used $5 \alpha$-cholestane as the internal standard and measured the concentrations of cholesterol, squalene, cholestenol, desmosterol, lathosterol (sterols reflecting cholesterol synthesis) ${ }^{(21)}$, cholestanol, campesterol, sitosterol, avenasterol (sterols reflecting cholesterol absorption) and sitostanol ${ }^{(21)}$. The confidence limit for variability percentage (n 20-29) was for cholesterol $3.9 \%$, cholestanol $4.4 \%$, desmosterol $7.1 \%$, lathosterol $5.0 \%$, campesterol $4.9 \%$ and sitosterol $3.0 \%$. 
Since the synthesis and absorption sterol ratios to cholesterol reflect the absolute whole-body synthesis and absorption percentage of cholesterol better than the concentrations ${ }^{(21)}$, all values were related to cholesterol obtained from the same GLC run, and the term ratio refers to the cholesterol-standardised values. To avoid extra decimals due to low concentrations of the non-cholesterol sterols, the ratios are multiplied by $100\left(10^{2} \mu \mathrm{mol} / \mathrm{mmol}\right.$ of cholesterol). Proportions of lathosterol/campesterol and lathosterol/sitosterol were also used as synthesis markers.

Total cholesterol, HDL and TAG in plasma were measured by an enzymatic method utilising cholesterol esterase, polyethylene glycol-modified cholesterol esterase and cholesterol oxidase, and TAG esterase and glycerol kinase, respectively, by a Modular P800 automatic analyser (Roche Diagnostics GmbH, Mannhein, Germany). The Friedewald formula ${ }^{(22)}$ was used in calculating the estimated concentration of LDL in plasma.

Carotenoids from infants' serum were analysed after deproteinisation with ethanol and extractions with hexane ${ }^{(23)}$. The supernatants were combined and evaporated to dryness and the residue dissolved in the mobile phase containing acetonitrile-methylene chloride-methanol (70:20:10, by volume). Separation of carotenoids was performed on a Nova-Pak C18 column (Waters, Dublin, Ireland), with a detection wavelength of $436 \mathrm{~nm}$ and a flow rate of $1 \mathrm{ml} / \mathrm{min}$. $\beta$-Carotene was quantified using ethyl $\beta$-apo- $\delta^{\prime}$-carotenoate as the internal standard. A stock solution of $\beta$-carotene was made by dissolving crystals in hexane and diluting in ethanol, the concentration of which was confirmed spectrophotometrically.

\section{Statistical analysis}

Values are reported as means and SD. The differences between the intervention and control groups were evaluated by the independent samples $t$ test for continuous variables, with the exception that the longitudinal analyses for mothers over pregnancy and breast-feeding and for infants' growth were made by repeated-measures ANOVA, where the baseline measurement was included as a covariate (analysis of covariance). When significant group differences were detected, the mean difference between the two groups with $95 \%$ CI over the follow-up period is presented. Posterior power analysis was conducted to evaluate the probability of the pilot study to find the observed difference in mothers' total cholesterol between groups statistically significant with a 0.05 two-sided significance level, and, subsequently, the required sample size was calculated to find the observed difference statistically significant for the purpose of both confirmatory and exploratory studies to be conducted in future. Significant correlations were analysed by Pearsons' correlation coefficient (r). All statistical analyses were performed on SPSS for Windows (version 13.0, SPSS Inc., Chicago, IL, USA).

\section{Results}

\section{Maternal serum lipids and sterols}

Of the sterols adjusted for cholesterol concentrations $\left(10^{2} \mu \mathrm{mol} /\right.$ mmol cholesterol; Table 1), the lathosterol ratio was 36.2 (SD 9.5-62.9) and that of sitostanol 2.6 (SD 1.1-4.1) higher over the follow-up, while the campesterol ratio was 49.8 (SD 8.7-90.9) lower over the follow-up in the serum of women in the intervention group compared with the controls. The proportions of lathosterol/campesterol and lathosterol/sitosterol were $0 \cdot 3(\operatorname{SD} 0 \cdot 1-0 \cdot 6)$ and $0 \cdot 7(\operatorname{SD~} 0 \cdot 2-1 \cdot 1)$ higher over the follow-up in the intervention group compared with the controls.

The desmosterol ratio increased over the pregnancy and continued to increase 1 month post-partum in both groups,

Table 1. Maternal serum lipids $(\mathrm{mmol} / \mathrm{l})$ and squalene and non-cholesterol sterols $\left(10^{2} \mu \mathrm{mol} / \mathrm{mmol}\right.$ cholesterol) in the intervention $(n+11)$ and control (n 10) groups

(Mean values and standard deviations)

\begin{tabular}{|c|c|c|c|c|c|c|c|c|c|c|c|c|c|}
\hline & \multicolumn{4}{|c|}{ First trimester } & \multicolumn{4}{|c|}{ Third trimester } & \multicolumn{4}{|c|}{1 month post-partum } & \multirow[b]{3}{*}{$P$ value* } \\
\hline & \multicolumn{2}{|c|}{ Intervention } & \multicolumn{2}{|c|}{ Control } & \multicolumn{2}{|c|}{ Intervention } & \multicolumn{2}{|c|}{ Control } & \multicolumn{2}{|c|}{ Intervention } & \multicolumn{2}{|c|}{ Control } & \\
\hline & Mean & SD & Mean & SD & Mean & SD & Mean & SD & Mean & SD & Mean & SD & \\
\hline Total cholesterol & 4.8 & 0.7 & $4 \cdot 8$ & 0.7 & $7 \cdot 0$ & 0.8 & $6 \cdot 6$ & $1 \cdot 1$ & 4.9 & 0.9 & 5.4 & 0.8 & 0.778 \\
\hline LDL- cholesterol† & $2 \cdot 4$ & 0.6 & $2 \cdot 4$ & 0.7 & $3 \cdot 7$ & 1.0 & $3 \cdot 7$ & 0.7 & 2.9 & 0.7 & $3 \cdot 3$ & 0.8 & 0.612 \\
\hline HDL- cholesterol & $2 \cdot 0$ & 0.3 & 1.9 & 0.4 & $2 \cdot 1$ & 0.3 & 1.9 & 0.3 & 1.8 & 0.3 & 1.8 & 0.4 & 0.660 \\
\hline TAG $†$ & 1.0 & 0.3 & $1 \cdot 1$ & 0.4 & $2 \cdot 2$ & 0.8 & $2 \cdot 3$ & 0.7 & 0.8 & 0.3 & 0.8 & 0.5 & 0.914 \\
\hline \multicolumn{14}{|c|}{ Estimates of cholesterol synthesis } \\
\hline Squalene & 14.9 & $2 \cdot 1$ & $14 \cdot 2$ & 4.7 & 11.4 & 3.1 & $14 \cdot 1$ & $7 \cdot 0$ & $15 \cdot 1$ & 5.4 & $13 \cdot 7$ & 4.2 & 0.668 \\
\hline Cholestenol & $15 \cdot 7$ & $4 \cdot 0$ & $21 \cdot 3$ & $6 \cdot 8$ & 17.5 & $2 \cdot 9$ & $17 \cdot 4$ & 4.4 & $17 \cdot 1$ & $5 \cdot 6$ & $16 \cdot 6$ & $5 \cdot 3$ & 0.451 \\
\hline Desmosterol† & $83 \cdot 6$ & $47 \cdot 4$ & $69 \cdot 6$ & $14 \cdot 8$ & $107 \cdot 2$ & $13 \cdot 4$ & $96 \cdot 2$ & $22 \cdot 3$ & $157 \cdot 0$ & 37.4 & $180 \cdot 6$ & $59 \cdot 6$ & 0.490 \\
\hline Lathosterol & $117 \cdot 8$ & $36 \cdot 6$ & $148 \cdot 3$ & 37.9 & $148 \cdot 3$ & 48.9 & $137 \cdot 7$ & 33.7 & $126 \cdot 4$ & 47.9 & 114.4 & 34.9 & 0.011 \\
\hline \multicolumn{14}{|c|}{ Estimates of cholesterol absorption } \\
\hline Cholestanol & $183 \cdot 8$ & 31.5 & $159 \cdot 6$ & $26 \cdot 7$ & $308 \cdot 0$ & $77 \cdot 7$ & 294.9 & $48 \cdot 1$ & 206.4 & 39.4 & $179 \cdot 3$ & 34.6 & 0.491 \\
\hline Campesterol & $307 \cdot 7$ & 63.6 & 257.7 & $95 \cdot 7$ & 221.4 & 58.4 & $238 \cdot 7$ & $85 \cdot 0$ & 240.5 & 63.8 & $255 \cdot 0$ & 89.2 & 0.020 \\
\hline Sitosterol & 141.4 & $36 \cdot 2$ & $120 \cdot 8$ & 38.0 & $111 \cdot 0$ & $32 \cdot 3$ & $115 \cdot 1$ & 33.3 & $116 \cdot 2$ & $36 \cdot 3$ & $128 \cdot 3$ & 39.9 & 0.070 \\
\hline Avenasterol & 38.6 & 8.7 & 34.9 & 8.3 & $35 \cdot 1$ & $9 \cdot 0$ & 33.5 & 4.9 & 39.4 & $7 \cdot 2$ & $36 \cdot 9$ & 5.5 & 0.567 \\
\hline Sitostanol & $9 \cdot 4$ & 3.3 & 8.9 & 1.4 & 11.4 & $2 \cdot 2$ & 8.9 & 1.9 & $12 \cdot 2$ & 3.8 & $9 \cdot 2$ & 1.9 & 0.002 \\
\hline Lathosterol/campesterol & 0.4 & 0.1 & 0.7 & 0.4 & 0.7 & 0.4 & 0.7 & 0.5 & 0.6 & 0.3 & 0.5 & 0.3 & 0.013 \\
\hline Lathosterol/sitosterol & 0.9 & 0.3 & 1.4 & 0.7 & 1.5 & 0.7 & 1.4 & 0.9 & $1 \cdot 2$ & 0.6 & 1.0 & 0.5 & 0.006 \\
\hline
\end{tabular}

ANCOVA, analysis of covariance.

${ }^{*}$ ANCOVA group effect.

† ANCOVA time effect $(P<0.05)$ 
the overall increase being 89.7 (SD 61.8-117.5) $10^{2} \mu \mathrm{mol} /$ mmol cholesterol. LDL- cholesterol and TAG increased by $1.3(\mathrm{SD} 0.9-1.8) \mathrm{mmol} / \mathrm{l}$ and 1.2 (SD 0.9-1.5) $\mathrm{mmol} / \mathrm{l}$, respectively, by the third trimester of pregnancy, after which their concentrations decreased by 0.7 (SD $0.3-1.1) \mathrm{mmol} / \mathrm{l}$ and 1.5 (SD $1.2-1 \cdot 7$ ) $\mathrm{mmol} / \mathrm{l}$, respectively, by 1 month post-partum in both groups. Mothers' serum total, LDL- or HDL- cholesterol or TAG did not differ between the intervention and control groups over the follow-up from the first trimester of pregnancy to 1 month post-partum (Table 1).

The analysis showed a non-significant treatment difference (intervention $v$. control) in the total cholesterol of $0.38(-0.47$ to 1.11$) \mathrm{mmol} / \mathrm{l}$ by the third trimester of pregnancy and -0.47 ( -1.17 to 0.24$) \mathrm{mmol} / \mathrm{l}$ by 1 month post-partum, providing 14 and $26 \%$ power, respectively, to detect the observed difference statistically significant. To find the observed differences statistically significant in future studies, the required sample size is 121 in each group during pregnancy and fifty-six in each group after pregnancy.

\section{Assessing the safety of maternal dietary intervention for the course of pregnancy and the infant}

Clinical characteristics. The pregnancies were uncomplicated and there were no differences in gestational weight gain between the intervention (15.7 (SD 4.1) $\mathrm{kg}$ ) and control $(15.7(\mathrm{SD} 6.8) \mathrm{kg})$ groups $(P=0.991)$. Equally, all infants were born full term $(40 \cdot 3$ (SD 1.1) and $40 \cdot 3$ (SD 1.3) weeks of gestation in the intervention and control groups, respectively, $P=0.974)$. The infants were exclusively breast-fed for 11 (SD 8) weeks (range 0-23 weeks) in the intervention group and for 16 (SD 2) weeks (range 13-20 weeks) in the control group $(P=0.08)$. Intervention had no influence on the infants' growth from birth up to the age of 12 months (Table 2). The mean height SD scores and weights proportional to heights were also within the population reference range in both groups ${ }^{(15)}$. Infants' cognitive development assessed on the Griffiths scale was equal in both groups at 12 months of

Table 2. Growth of infants from birth to 12 months of age according to mother's dietary intervention or control group

(Mean values and standard deviations)

\begin{tabular}{|c|c|c|c|c|}
\hline & \multicolumn{2}{|c|}{$\begin{array}{l}\text { Intervention* } \\
\quad(n 11)\end{array}$} & \multicolumn{2}{|c|}{$\begin{array}{l}\text { Control } \\
(n 10)\end{array}$} \\
\hline & Mean & SD & Mean & SD \\
\hline \multicolumn{5}{|l|}{ At birth } \\
\hline Height (SD scores) & 0.2 & 0.8 & 0.6 & $1 \cdot 1$ \\
\hline Weight (\% for height) & -5.9 & $7 \cdot 5$ & $0 \cdot 1$ & $14 \cdot 5$ \\
\hline \multicolumn{5}{|l|}{ At 1 month of age } \\
\hline Height (SD scores) & 0.5 & 0.6 & 0.9 & 0.8 \\
\hline Weight (\% for height) & $1 \cdot 3$ & $9 \cdot 2$ & $2 \cdot 3$ & $8 \cdot 8$ \\
\hline \multicolumn{5}{|l|}{ At 6 month of age $†$} \\
\hline Height (SD scores) & $0 \cdot 2$ & 0.7 & $0 \cdot 1$ & 0.6 \\
\hline Weight (\% for height) & -3.9 & $7 \cdot 8$ & $2 \cdot 8$ & $10 \cdot 9$ \\
\hline \multicolumn{5}{|l|}{ At 12 month of age } \\
\hline Height (SD scores) & 0.3 & 0.7 & $0 \cdot 1$ & 0.7 \\
\hline Weight (\% for height) & -4.4 & $9 \cdot 0$ & 0.1 & $10 \cdot 5$ \\
\hline
\end{tabular}

age (164 (SD 9) in the intervention group, $n$ 11, and 162 (SD 6) in the control group, $n 8 ; P=0.701$ ).

Breast-milk and infants' serum lipids and sterols. In breast milk, no significant differences were observed in total cholesterol, but the desmosterol ratio was lower and the campesterol ratio tended to be lower in the intervention group compared with the controls (Table 3). No significant differences according to mothers' group were detected in lipids or sterols in infants' serum either at 1 or 6 month of age (Table 4). For evaluation of associations between estimates for mothers and infants, the intervention and control groups were combined. Mothers' serum cholestenol, desmosterol, lathosterol, campesterol and sitosterol were associated with those of breast milk but not with the infants' serum (Table 5). Likewise, no association was observed between breast-milk and infants' serum sterols.

Infants' serum $\beta$-carotene. No significant differences in mean $\beta$-carotene in infants' serum at 1 month 144.6 (SD 121.8) v. $227.8(\mathrm{SD} \mathrm{103.4)} \mathrm{nmol} / \mathrm{l}, P=0.117)$ and 6 months (1663.3 (SD 1359.6) v. 2465 (SD 1975.0) nmol/l, $P=0.335)$ of age were detected between the intervention and control groups. When the results were adjusted for cholesterol, the serum $\beta$-carotene in infant serum was lower in the intervention group at 1 month of age compared with the controls (29.1 (SD 18.6) v. 59.3 (SD 22.4) nmol/mmol cholesterol, $P=0.006)$, while no significant difference was detected between the groups at 6 months of age (460.5 (SD 371.9) $v$. $598 \cdot 3$ (SD 400·0), nmol $/ \mathrm{mmol}$ cholesterol, $P=0 \cdot 477$; Fig. 1).

\section{Discussion}

The present study is the first to evaluate the impact of plant stanol ester spread consumption on both mothers and infants during pregnancy and breast-feeding. The consumption of plant stanols was reflected in the markers of cholesterol synthesis and absorption in the mothers' serum. Importantly, no adverse effects on either the mother or the infant were detected, as indicated by length of gestation, birth weight and growth in infancy, with the exception of lower cholesterol-adjusted $\beta$-carotene concentrations at the age of 1 month in infants, whose mothers had consumed plant stanols. The results offer a novel and possibly safe tool for controlling

Table 3. Breast-milk total cholesterol (mmol/l) and squalene and noncholesterol sterols $\left(10^{2} \mu \mathrm{mol} / \mathrm{mmol}\right.$ cholesterol) from mothers 1 month post-partum in the intervention $(n 11)$ and the control groups $(n 10)^{(1)}$ (Mean values and standard deviations)

\begin{tabular}{|c|c|c|c|c|c|}
\hline & \multicolumn{2}{|c|}{ Intervention } & \multicolumn{2}{|c|}{ Control } & \multirow[b]{2}{*}{$P$ value ${ }^{*}$} \\
\hline & Mean & SD & Mean & SD & \\
\hline Total cholesterol & 0.4 & 0.1 & 0.4 & 0.1 & 0.365 \\
\hline Squalene & $2499 \cdot 9$ & $891 \cdot 7$ & $2918 \cdot 1$ & 924.9 & 0.305 \\
\hline Cholestenol & $43 \cdot 1$ & $10 \cdot 3$ & $50 \cdot 1$ & $10 \cdot 2$ & 0.133 \\
\hline Desmosterol & $10703 \cdot 7$ & $3661 \cdot 3$ & 13821.9 & 2607.9 & 0.038 \\
\hline Lathosterol & $255 \cdot 7$ & $93 \cdot 1$ & $283 \cdot 7$ & $97 \cdot 6$ & 0.509 \\
\hline Cholestanol & $340 \cdot 3$ & $53 \cdot 0$ & $329 \cdot 5$ & $25 \cdot 5$ & 0.554 \\
\hline Campesterol & 308.5 & $74 \cdot 2$ & 371.8 & $68 \cdot 2$ & 0.056 \\
\hline Sitosterol & $95 \cdot 7$ & $33 \cdot 2$ & $116 \cdot 1$ & $37 \cdot 8$ & 0.203 \\
\hline Avenasterol & $252 \cdot 6$ & $81 \cdot 5$ & $300 \cdot 2$ & $37 \cdot 5$ & 0.107 \\
\hline Sitostanol & $79 \cdot 8$ & $16 \cdot 8$ & 83.5 & $23 \cdot 7$ & 0.683 \\
\hline
\end{tabular}

*Independent samples $t$ test. 
Table 4. Infants' serum lipids $(\mathrm{mmol} / \mathrm{l})$ and squalene and non-cholesterol sterols $\left(10^{2} \mu \mathrm{mol} / \mathrm{mmol}\right.$ cholesterol) at 1 and 6 months of age in the intervention ( $n$ 10) and the control groups $(n 10)$

(Mean values and standard deviations)

\begin{tabular}{|c|c|c|c|c|c|c|c|c|}
\hline & \multicolumn{4}{|c|}{1 month } & \multicolumn{4}{|c|}{6 months } \\
\hline & \multicolumn{2}{|c|}{ Intervention* } & \multicolumn{2}{|c|}{ Control } & \multicolumn{2}{|c|}{ Intervention* } & \multicolumn{2}{|c|}{ Control† } \\
\hline & Mean & SD & Mean & SD & Mean & SD & Mean & SD \\
\hline Total cholesterol ${ }^{(3)}$ & $3 \cdot 7$ & 0.5 & $3 \cdot 6$ & 0.6 & 3.6 & 0.9 & $3 \cdot 8$ & 0.5 \\
\hline HDL- cholesterol ${ }^{(3)}$ & 1.4 & 0.4 & 1.4 & 0.4 & 1.2 & 0.3 & $1 \cdot 2$ & 0.3 \\
\hline \multicolumn{9}{|c|}{ Estimates of cholesterol synthesis } \\
\hline Squalene & 36.5 & 11.5 & 32.4 & 10.5 & $27 \cdot 2$ & 13.0 & $21 \cdot 6$ & $5 \cdot 1$ \\
\hline Cholestenol & $26 \cdot 7$ & $21 \cdot 3$ & $29 \cdot 3$ & $29 \cdot 3$ & 21.6 & $6 \cdot 7$ & $19 \cdot 6$ & $4 \cdot 1$ \\
\hline Desmosterol & $242 \cdot 1$ & 31.5 & $269 \cdot 0$ & $42 \cdot 8$ & $145 \cdot 4$ & $38 \cdot 8$ & $161 \cdot 3$ & $29 \cdot 3$ \\
\hline Lathosterol & 85.4 & $22 \cdot 7$ & 84.5 & $12 \cdot 3$ & 104.6 & $34 \cdot 3$ & $99 \cdot 0$ & 24.3 \\
\hline \multicolumn{9}{|c|}{ Estimates of cholesterol absorption } \\
\hline Cholestanol & $190 \cdot 8$ & $22 \cdot 2$ & 205.9 & $32 \cdot 4$ & 160.5 & 19.9 & $166 \cdot 6$ & $16 \cdot 4$ \\
\hline Campesterol & 69.4 & 34.8 & 44.9 & $19 \cdot 3$ & $145 \cdot 3$ & $89 \cdot 3$ & 88.9 & $55 \cdot 2$ \\
\hline Sitosterol & $30 \cdot 2$ & $20 \cdot 8$ & $15 \cdot 5$ & 7.7 & $95 \cdot 8$ & $59 \cdot 3$ & $65 \cdot 1$ & 37.3 \\
\hline Avenasterol & $18 \cdot 0$ & 3.8 & $15 \cdot 4$ & 3.9 & 41.9 & $17 \cdot 1$ & 33.6 & $12 \cdot 9$ \\
\hline Sitostanol & $8 \cdot 1$ & 3.5 & $7 \cdot 7$ & $3 \cdot 1$ & $11 \cdot 7$ & $3 \cdot 1$ & $10 \cdot 7$ & 1.9 \\
\hline Lathosterol/campesterol & 1.5 & 0.8 & $2 \cdot 1$ & 0.6 & 1.3 & 1.5 & 1.4 & 0.7 \\
\hline Lathosterol/sitosterol & 4.6 & 3.9 & 6.5 & $2 \cdot 6$ & 2.5 & 3.4 & 1.9 & 0.9 \\
\hline
\end{tabular}

*All differences between groups at 1 and 6 months of age NS, independent samples $t$ test.

†The number of subjects are seven to nine due to insufficient sample for analysis or discontinuation.

blood lipids in hypercholesterolaemic women during pregnancy, with a potential to benefit not only the mother but also the child ${ }^{(3,4)}$, and provide power analyses for future confirmatory and exploratory studies. As plant stanol ester spreads are not currently recommended during pregnancy, further research on their merits and risks including their lipid-lowering effects in cases of hypercholesterolaemia in pregnancy is to be encouraged.

In the present study, the consumption of plant stanol ester spreads was reflected in mothers' serum non-cholesterol sterols in such a way that markers for cholesterol synthesis, the ratio of lathosterol to cholesterol and the ratios of lathosterol to campesterol and lathosterol to sitosterol were increased and the marker for cholesterol absorption, campesterol, was reduced. The serum sitostanol ratio was increased due to the consumption of stanol ester margarine, but the levels remained low. However, no impact on serum lipoproteins was detected. This is unlikely to be due to the small dose of plant stanols consumed by the subjects, bearing in mind that the appropriate dose for lipid lowering in pregnancy has not been studied, as a similar dose has previously been shown to reduce blood cholesterol concentrations significantly ${ }^{(24,25)}$ and customary use of low doses has been shown to stabilise cholesterol levels ${ }^{(26)}$. Another possible mechanism might be that by reason of cholesterol homeostasis, when cholesterol absorption is inhibited, cholesterol synthesis was up-regulated, thus interfering with the serum cholesterol lowering. An alternative and very likely explanation may be that the physiological adaptations occurring during pregnancy obscure the impact of changes in dietary composition, including the consumption of stanols at this level. Moreover, the small population studied in the present pilot study resulted in insufficient power to show differences between the groups in cholesterol and lipoproteins, the baseline levels of which were low. Indeed, a recent study has shown that baseline serum lipoprotein concentrations are related to plant stanol-induced changes, with higher baseline

Table 5. Correlations between total cholesterol and squalene and non-cholesterol sterols in mothers' and infants' serum and breast milk*

\begin{tabular}{|c|c|c|c|c|c|c|}
\hline & \multicolumn{2}{|c|}{$\begin{array}{l}\text { Mothers' serum with } \\
\text { infants' serum }\end{array}$} & \multicolumn{2}{|c|}{$\begin{array}{l}\text { Mothers' serum with } \\
\text { breast milk }\end{array}$} & \multicolumn{2}{|c|}{$\begin{array}{l}\text { Breast milk with infants' } \\
\text { serum }\end{array}$} \\
\hline & $R(n 19)$ & $P$ value & $R(n 20)$ & $P$ value & $R(n 20)$ & $P$ value \\
\hline Total cholesterol & -0.124 & 0.612 & 0.179 & 0.449 & 0.234 & 0.321 \\
\hline Squalene & 0.335 & $0 \cdot 161$ & 0.082 & $0 \cdot 730$ & 0.017 & 0.943 \\
\hline Cholestenol & -0.101 & 0.680 & 0.528 & 0.017 & -0.048 & 0.842 \\
\hline Desmosterol & 0.416 & 0.076 & 0.623 & 0.003 & 0.306 & 0.189 \\
\hline Lathosterol & -0.051 & 0.836 & 0.593 & 0.006 & -0.082 & 0.730 \\
\hline Cholestanol & -0.321 & $0 \cdot 180$ & 0.329 & $0 \cdot 157$ & 0.079 & 0.739 \\
\hline Campesterol & 0.307 & $0 \cdot 201$ & 0.759 & $<0.001$ & 0.283 & 0.226 \\
\hline Sitosterol & 0.283 & $0 \cdot 241$ & 0.552 & 0.012 & 0.216 & 0.361 \\
\hline Avenasterol & 0.416 & 0.077 & -0.062 & 0.795 & -0.404 & 0.077 \\
\hline Sitostanol & -0.093 & $0 \cdot 706$ & 0.086 & 0.719 & 0.072 & 0.763 \\
\hline
\end{tabular}

${ }^{*}$ All analyses were completed at the infants' age of 1 month. 


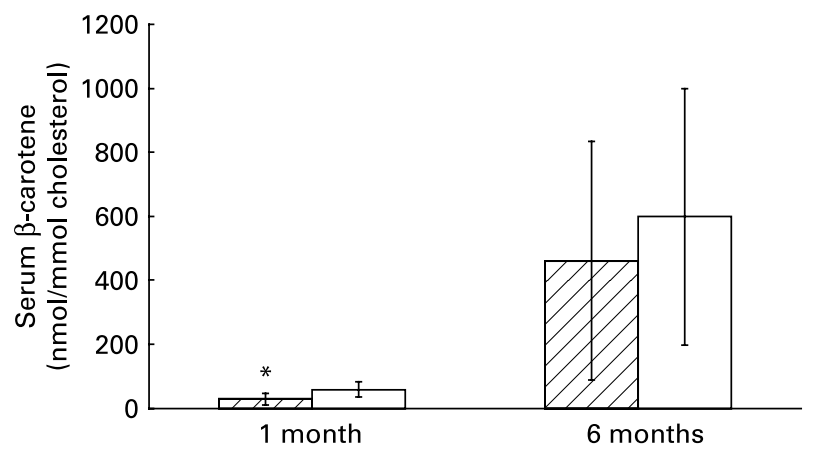

Fig. 1. Serum $\beta$-carotene concentration $(\mathrm{nmol} / \mathrm{mmol}$ cholesterol) in infants at the age of 1 and 6 months according to mother's dietary intervention (四) or control $(\square)$ group. Data are presented as means and standard deviations. * Statistically significantly different from controls, independent samples $t$ test, $P=0.006$.

levels resulting in larger decreases ${ }^{(27)}$. Importantly, indicated by the present results, the means for interfering with blood lipid levels during pregnancy are limited, but the case was different already 1 month after delivery. The intervention resulted in a lower blood cholesterol by an average of $0.47 \mathrm{mmol} / 1$ with $26 \%$ power to detect the difference statistically significant. The study also provided a base for feasible further studies, with fifty-six subjects per group being sufficient to detect the observed difference statistically significant. Undeniably, with anticipated beneficial effects for both the mother and the child of the regulation of blood lipids ${ }^{(3,4)}$, further studies applying the calculated sample size may clarify the issues of appropriate dose and duration of consuming plant stanols with most likely beneficial effects seen in mothers manifesting with hypercholesterolaemia before pregnancy or excessive rise in blood cholesterol during pregnancy.

Concerns may be raised over perturbation of cholesterol metabolism during pregnancy and lactation by stanol consumption with risks understandably envisaged for both mothers and infants: the rise in maternal serum cholesterol concentration during pregnancy is considered physiological ${ }^{(10)}$; cholesterol is essential for fetal development ${ }^{(28)}$ and is secreted in breast milk $^{(29)}$; finally, the absorption of fat-soluble vitamins may be disturbed ${ }^{(25,30-32)}$. Thus, plant stanol ester products are not currently recommended during pregnancy or lactation. The present data, however, do not support these conceptions as, firstly, a rise in blood lipoproteins and TAG in late pregnancy was observed, as indeed previously reported ${ }^{(5-7)}$, regardless of plant stanol ester consumption. Although the rise appears to be associated with physiological hormonal changes in pregnancy ${ }^{(10)}$, there is evidence suggesting that this increase in blood lipids may be related to complications during pregnancy ${ }^{(4)}$ and the development of cardiovascular lesions in child ${ }^{(3)}$. Thus, to prevent these undesirable outcomes, further research with plant stanols is necessitated. Secondly, although cholesterol is known to be essential for the developing fetus, experimental studies showing that prevention of cholesterol synthesis in pregnant rats results in anomalies in the offspring ${ }^{(28)}$, there is no evidence that hindering cholesterol absorption within the gastrointestinal tract by plant stanol consumption would hamper hepatic cholesterol synthesis. Instead, normal pregnancy and lactation are associated with increased cholesterol synthesis ${ }^{(33)}$, an observation also supported by those in the present study, since the ratio of lathosterol increased during pregnancy and lactation regardless of whether stanol ester spreads were consumed or not. However, desmosterol in breast milk was lower in the women from the intervention group, possibly suggesting a lower rate of breast cholesterol synthesis in mothers consuming plant stanols, but the significance of this remains unclear as no other differences were detected in breast-milk sterols or cholesterol in mothers consuming stanols or not, nor differences in infants' serum cholesterol or growth and development. No disturbance to the infants' cholesterol metabolism by maternal intervention was detected. Furthermore, although mothers' serum and breast-milk sterols were associated, no relations between mothers' serum or breast-milk and infants' serum cholesterol or sterols emerged, suggesting that the breast-fed infants' cholesterol synthesis and absorption are relatively independent of those in the mother. Consumption of plant stanols fairly consistently increases their serum levels ${ }^{(21)}$. Despite the fact that no changes were seen in mothers' serum or milk, or infants' serum, an accumulation of plant stanols in the tissues is not excluded. However, additional support for the safety of consuming plant stanols during pregnancy and lactation is derived from the clinical data. Maternal plant stanol consumption had no impact on either the length of gestation or the infants' growth over the first year, nor was there any difference between groups in infants' cognitive development at 12 months of age, and all scores were considered normal ${ }^{(16)}$. This safety aspect is further emphasised by the results showing an association of highcholesterol levels in early pregnancy with an increased risk of preterm birth ${ }^{(34)}$. Unfavourable levels of lipoproteins have also been associated with large birth weight, another disadvantageous outcome of pregnancy ${ }^{(35)}$.

Finally, we evaluated the impact of intervention on the infants' serum $\beta$-carotene concentration, as plant stanol consumption has previously been related to their reduced levels ${ }^{(25,30-32)}$, although results to the contrary have been obtained $^{(36)}$. We observed no differences in absolute serum $\beta$-carotene concentrations at the infants' age of 1 and 6 months, but cholesterol-adjusted serum $\beta$-carotene at 1 month of age was lower in the intervention group. As the difference was observed only when adjusted for cholesterol, it may indicate that intervention infants had elevated cholesterol synthesis, although the markers for cholesterol synthesis or absorption in infant serum did not differ significantly between the groups. Notably, the serum $\beta$-carotene concentrations were low at 1 month of age and were raised at 6 months in all children regardless of maternal stanol consumption, this probably reflecting the intake of $\beta$-carotene from complementary foods. The significance of the infants' reduced $\beta$-carotene concentration remains to be elucidated, taking into consideration that low levels of $\beta$-carotene have also previously been measured in infants with no dietary intervention $^{(37)}$. The results of the present pilot study showing the impact of plant stanol ester spread consumption on markers of cholesterol synthesis and absorption in mothers' serum and importantly clinical safety encourage further studies, on the one hand, in at-risk populations for the management of hyperlipidaemia during pregnancy and lactation, and, on the other hand, in a larger general population, bearing in mind the risks related to an increase in blood lipids during 
pregnancy $^{(3,4,34)}$. Furthermore, the recent discussion on the management of hypercholesterolaemia in children ${ }^{(38)}$ reinforces the development of dietary therapy, possibly with plant stanols, for the regulation of blood cholesterol, particularly to eliminate the potential risks attested to medication. If plant stanol ester spreads are consumed, care should be taken to ensure a balanced dietary intake and, particularly, an increased intake of carotenoids in view of previous results showing that sufficient dietary intake prevents the decline in carotenoids accompanying consumption of stanols ${ }^{(14)}$.

\section{Acknowledgements}

Financial support from the Academy of Finland, the Social Insurance Institution of Finland, provision of spreads by Raisio plc and revision of the English text by Mr Robert MacGilleon are greatly appreciated. The authors declare that there is no personal or financial conflict of interest associated with the present manuscript. K. L. and E. I. were responsible for the design of the study and organisation of data collection. L. K., H. G. and T. A. M. were responsible for analysing non-cholesterol sterols. K. L., E. I., H. G. and T. A. M. contributed to the interpretation of the results and the writing and revising of the manuscript.

\section{References}

1. Gylling H \& Miettinen TA (2001) A review of clinical trials in dietary interventions to decrease the incidence of coronary artery disease. Curr Control Trials Cardiovasc Med 2, $123-128$.

2. Schaefer EJ (2002) Lipoproteins, nutrition, and heart disease. Am J Clin Nutr 75, 191-212.

3. Napoli C, Glass CK, Witztum JL, et al. (1999) Influence of maternal hypercholesterolaemia during pregnancy on progression of early atherosclerotic lesions in childhood: fate of early lesions in children (FELIC) study. Lancet 354, 1234-1241.

4. Solomon CG, Carroll JS, Okamura K, et al. (1999) Higher cholesterol and insulin levels in pregnancy are associated with increased risk for pregnancy-induced hypertension. Am $J$ Hypertens 12, 276-282.

5. Montelongo A, Lasunción MA, Pallardo LF, et al. (1992) Longitudinal study of plasma lipoproteins and hormones during pregnancy in normal and diabetic women. Diabetes $\mathbf{4 1}$, 1651-1659.

6. Brizzi P, Tonolo G, Esposito F, et al. (1999) Lipoprotein metabolism during normal pregnancy. Am J Obstetr Gynecol 181, 430-434.

7. Martin U, Davies C, Hayavi S, et al. (1999) Is normal pregnancy atherogenic? Clin Sci 96, 421-425.

8. Catalano PM, Tyzbir ED, Roman NM, et al. (1991) Longitudinal changes in insulin release and insulin resistance in nonobese pregnant women. Am J Obstet Gynecol 165, 1667-1672.

9. Herrera E, Lasuncion MA, Gomez-Coronado D, et al. (1988) Role of lipoprotein lipase activity on lipoprotein metabolism and the fate of circulating triglycerides in pregnancy. Am J Obstet Gynecol 158, 1575-1583.

10. Butte NF (2000) Carbohydrate and lipid metabolism in pregnancy: normal compared with gestational diabetes mellitus. Am J Clin Nutr 71, 1256-1261.

11. McMurry M, Connor WE \& Goplerud CP (1981) The effects of dietary cholesterol upon the hypercholesterolemia of pregnancy. Metabolism 30, 869-879.
12. Katan MB, Grundy SM, Jones P, et al. (2003) Efficacy and safety of plant stanols and sterols in the management of blood cholesterol levels. Mayo Clin Proc 78, 965-978.

13. Phillips KM, Tarrago-Trani MT \& Stewart KK (1999) Phytosterol content of experimental diets differing in fatty acid composition. Food Chem 64, 415-422.

14. Noakes M, Clifton P, Ntanios F, et al. (2002) An increase in dietary carotenoids when consuming plant sterols or stanols is effective in maintaining plasma carotenoid concentrations. Am J Clin Nutr 75, 79-86.

15. Sorva R, Perheentupa J \& Tolppanen EM (1984) A novel format for growth chart. Acta Paediatr 73, 527-529.

16. Griffiths R (1970) The Abilities of Babies. London: University of London Press.

17. Nordic Working Group on Diet and Nutrition (1996) Nordic nutrition recommendations. Scand J Nutr 40, 161-165.

18. Becker W, Lyhne N, Pedersen AN, et al. (2004) Nordic nutrition recommendations 2004 - integrating nutrition and physical activity. Scand J Nutr 48, 178-187.

19. Piirainen T, Isolauri E, Lagström H, et al. (2006) Impact of dietary counseling on nutrient intake during pregnancy: a prospective cohort study. Brit J Nutr 96, 1095-1104.

20. Miettinen TA (1988) Cholesterol metabolism during ketoconazole treatment in man. $J$ Lipid Res 29, 43-51.

21. Miettinen TA, Tilvis RS \& Kesäniemi YA (1990) Serum plant sterols and cholesterol precursors reflect cholesterol absorption and synthesis in volunteers of a randomly selected male population. Am J Epidemiol 131, 20-31.

22. Friedewald WT, Levy RI \& Fredrickson D (1972) Estimation of the concentration of low-density lipoprotein cholesterol in plasma, without use of the preparative ultracentrifuge. Clin Chem 18, 499-502.

23. Craft NE, Brown ED \& Smith JC (1988) Effects of storage and handling conditions on concentrations of individual carotenoids, retinol, and tocopherol in plasma. Clin Chem 34, 44-48.

24. Hallikainen MA, Sarkkinen ES \& Uusitupa MIJ (2000) Plant stanol esters affect serum cholesterol concentrations of hypercholesterolemic men and women in a dose-dependent manner. J Nutr 130, 767-776.

25. Tammi A, Rönnemaa T, Gylling H, et al. (2000) Plant stanol ester margarine lowers serum total and low-density lipoprotein cholesterol concentrations of healthy children: the STRIP project. J Pediatr 136, 503-510.

26. Wolfs M, de Jong N, Ocke MC, et al. (2006) Effectiveness of customary use of phytosterol/-stanol enriched margarines on blood cholesterol lowering. Food Chem Toxicol 44, 1682-1688.

27. Naumann E, Plat J, Kester AD, et al. (2008) The baseline serum lipoprotein profile is related to plant stanol induced changes in serum lipoprotein cholesterol and triacylglycerol concentrations. J Am Coll Nutr 27, 117-126.

28. Roux C, Wolf C, Mulliez N, et al. (2000) Role of cholesterol in embryonic development. Am J Clin Nutr 71, 1270-1279.

29. Rodriguez-Palmero M, Koletzko B, Kunz C, et al. (1999) Nutritional and biochemical properties of human milk, part 2: lipids, micronutrients, and bioactive factors. Clin Perinatol 26, 335-359.

30. Gylling H, Puska P, Vartiainen E, et al. (1999) Retinol, vitamin $\mathrm{D}$, carotenes and $\alpha$-tocopherol in serum of a mildly hypercholesterolemic population treated with sitostanol ester margarine. Atherosclerosis 145, 279-285.

31. Hallikainen MA, Sarkkinen ES \& Uusitupa MIJ (1999) Effects of low-fat stanol ester enriched margarines on concentrations of serum carotenoids in subjects with elevated serum cholesterol concentrations. Eur J Clin Nutr 53, 966-969.

32. Plat J, van Onselen ENM, van Heugten MMA, et al. (2000) Effects on serum lipids, lipoproteins and fat soluble antioxidant concentrations of consumption frequency of margarines and 
shortenings enriched with plant stanol esters. Eur J Clin Nutr 54, 671-677.

33. Nikkilä K, Riikonen S, Lindfors M, et al. (1996) Serum squalene and noncholesterol sterols before and after delivery in normal and cholestatic pregnancy. J Lipid Res 37, 2687-2695.

34. Catov JM, Bodnar LM, Kip EK, et al. (2007) Early pregnancy lipid concentrations and spontaneous preterm birth. Am J Obstetr Gynecol 197, 610e1-610e7.

35. Romundstad PR, Smith GD, Nilsen TIL, et al. (2007) Associations of prepregnancy cardiovascular risk factors with the oppspring's birth weight. Am J Epidemiol 166, 1359-1364.
36. Hallikainen MA \& Uusitupa MIJ (1999) Effects of 2 low-fat stanol ester-containing margarines on serum cholesterol concentrations as part of a low-fat diet in hypercholesterolemic subjects. Am J Clin Nutr 69, 403-410.

37. Ostrea EM, Balun JE, Winkler R, et al. (1986) Influence of breast-feeding on the restoration of the low serum concentration of vitamin $\mathrm{E}$ and beta-carotene in the newborn infant. Am J Obstetr Gynecol 154, 1014-1017.

38. de Ferranti S \& Ludwig DS (2008) Storm over statins - the controversy surrounding pharmacologic treatment of children. $N$ Engl J Med 359, 1309-1312. 